\title{
Efecto hipoglucemiante del consumo de yacón (Smallantus sonchifolius) en ratones diabéticos tipo 2 inducidos con aloxano
}

\author{
Hypoglycemic effect of consumption of yacon (yacón) in type 2 diabetic mice induced with alloxan
}

América Mejía Vásquez* ${ }^{*}$ Delcy Zuloeta Guerrero², Félix Palacios Morales ${ }^{3}$

\section{RESUMEN}

\begin{abstract}
Objetivo: Determinar la efectividad del consumo de yacón (Smallantus sonchifolius) sobre los niveles de glucosa en ratones albinos con diabetes mellitus tipo 2 inducidos con aloxano. Material y métodos: La muestra estuvo conformada por un grupo control y tratamiento con 21 ratones albinos en cada caso, con pesos promedios entre 30-40g, ejecutados en el laboratorio de anatomía y microscopia. Los ratones fueron inducidos con Aloxano en solución de $0.5 \mathrm{gr} / 200 \mathrm{ml}$ de agua destilada administrados por vía intraperitoneal en dosis de 0,1 a 0,7 ml/ 36 días, para inducir a DM2. La evaluación de glucosa basal promedio fue de $110 \mathrm{mg} / \mathrm{dl}$ para control y tratamiento. Resultados: en el grupo con tratamiento el nivel de glucosa inicial inducida fue $135.30 \mathrm{mg} / \mathrm{dl}$, redujo a $107.70 \mathrm{mg} /$ dl, a un nivel de confianza al 99\%, presentó diferencia estadística significativa respecto al valor tabulado de $(\mathrm{p}<01)$. La glucosa inicial y final del control fue $130.60 \mathrm{mg} / \mathrm{dl}$ y $151.45 \mathrm{mg} / \mathrm{dl}$ respectivamente, no tuvo grado de significancia. Conclusiones: El estudio anatomopatológico por efecto citotóxico del Aloxano en el páncreas, presentó malformación de células estructurales y destrucción de los islotes de Langerhans, además el consumo de $100 \mathrm{~g}$ de yacón en ratones albinos inducidos a DM2 durante un periodo de 34 días disminuyó significativamente el nivel de glicemia.
\end{abstract}

Palabras clave: Yacón, Páncreas, islotes de Langerhans, Aloxano, ratones albinos.

\begin{abstract}
Objective: Determine the effectiveness of the consumption of yacon (Smallantus sonchifolius) on glucose levels in diabetes mellitus type 2 with alloxan-induced albino mice. Methods: The sample was comprised of a control group and treatment with 21 albino mice in each case, with weights averages between 30 - 40g, executed in the laboratory of Anatomy and microscopy. The mice were induced with Alloxan in solution of $0.5 \mathrm{gr} / 200 \mathrm{ml}$ of distilled water administered intraperitoneally at doses of 0.1 to $0.7 \mathrm{ml} / 36$ days to induce to DM2. The average evaluation of basal glucose was $110 \mathrm{mg} / \mathrm{dl}$ for control and treatment. Results: In the treatment group induced initial glucose levels was $135.30 \mathrm{mg} / \mathrm{dl}$, reduced to $107,70 \mathrm{mg} / \mathrm{dl}$, with a confidence level of $99 \%$, presented significant statistical differences in the tabulated value of $(p<01)$. The initial glucose and end of the control group was $130.60 \mathrm{mg} / \mathrm{dl}$ and $151.45 \mathrm{mg} / \mathrm{dl}$, respectively, had no degree of significance. Conclusions: the study anatomy pathological by cytotoxic effect of Alloxan on the pancreas, presented malformation of structural cells and destruction of the islets of Langerhans, also the consumption of $100 \mathrm{~g}$ of yacon in albino mice induced to DM2 during a period of 34 days significantly decreased the level of glycemia.
\end{abstract}

Keywords: Yacon, Pancreas, islets of Langerhans, Alloxan, albino mice

\footnotetext{
${ }^{1}$ Bachiller en Nutrición Humana, Clínica Good Hope, Miraflores, Lima, Perú

${ }^{2}$ Bachiller en Nutrición Humana, Facultad de Ciencias de la Salud, Universidad Peruana Unión.

${ }^{3}$ Jefe de Laboratorio de Biología, Docente de la E.A.P de Nutrición Humana, Universidad Peruana Unión.
} 


\section{INTRODUCCIÓN}

La Federación internacional de la diabetes (FDI), indica que en todo el mundo existen 382 millones de personas que viven con diabetes y más de 316 millones sufren tolerancia anormal de la glucosa. Asimismo, se considera que para el 2035, la cifra de diabéticos ascienda a 592 millones (1 de cada diez); cabe mencionar que más del $50 \%$ de las personas que viven con diabetes no lo saben (Hirst M, Williams R, 2014) (Aschener P, 2013).

En el Perú se ha demostrado alrededor de 2 millones de personas afectadas siendo el 90\% de la DMT 2 y es la décimo quinta causa de muerte. La prevalencia se estimada de 5,4\% en el año 2013. La magnitud de la misma está en aumento debido al incremento de la obesidad, el sobrepeso, el sedentarismo y los hábitos inadecuados de alimentos (Portugal W, 2015) (Jurupe H; Herencia V; Robles E, 2007). El mayor porcentaje de personas con DMT2 se ubica en Lima Metropolitana con un 7.6\% (Gonzales N, Rodríguez E, 2013), y distribuido en la costa un $3.4 \%$, en la selva $2.5 \%$ y en la sierra un 2\% (Bash, 2015). Las estadísticas hacen referencias a la situación alarmante en las que se ubica esta enfermedad considerada como una de las primeras cinco causas de muerte a nivel mundial (Claude, 2011). Esta enfermedad, se caracteriza por la insuficiencia absoluta o relativa de la secreción de insulina por el páncreas y por la resistencia de la misma en los tejidos, ocasionando un desequilibrio del metabolismo de carbohidratos, lípidos y proteínas (Gonzales R, 2000) (Aguilar C, Loreto M, 2013).

También conducen a una hiperglucemia y muchas complicaciones tales como: enfermedades cardiovasculares, enfermedad renal, neuropatías diabéticas, amputaciones no debida a traumas, cegueras, enfermedades infecciosas, infartos cerebrales, incapacidad con prematuras y daños colaterales del tratamiento farmacológico hipoglucemiante alternativa inferiores (Isla, 2012) (Ziaroyska J, Fernandez E, Millela L, 2013).

Aunque las terapias tradicionales como las inyecciones de insulina son eficaces en el control de glucemia, presentan algunos efectos secundarios y limitaciones en ciertas poblaciones. Las causas y el control de la DM2 no están claras. Pero hay una alta evidencia para afirmar que los factores dietéticos están implicados en su regulación y prevención.

La Organización Mundial de Salud(OMS) ha estimado que gran porcentaje de la población mundial utiliza la medicina tradicional para satisfacer sus necesidades de atención primaria de salud y que gran parte de los tratamientos implica el uso de extractos de plantas o sus principios activos glucémico(Gordillo, rocha,
2009). Adicionalmente, se muestra una demanda del descubrimiento de alimentos funcionales, especialmente para el tratamiento de enfermedades no transmisibles como la diabetes (Carla et al., 2016) (Ferreiras S, Azevedo C, Vardanega P, 2013).Dentro de esto alimentos funcionales podemos mencionar el yacón (Valentová K, 2003). Que es un fruto natural que contiene muchos beneficios en la salud, que gran parte de sus propiedades se deben a los fructooligosacáridos (Oliveira et al., 2013) (Ziaroyska J, Fernandez E, Millela L, 2013).

El estudio estará centrado en la utilización de la raíces de yacon, que pertenece a la familia Asteraceae, servirá para comprobar sus propiedades hipoglucemiantes aplicados a pacientes con este tipo de trastorno, para la cual serán administrados la raíz y las hojas en el tratamiento de la diabetes y otros trastornos metabólicos relacionados. Siendo el objetivo determinar la efectividad del consumo de yacón (Smallanthus sonchifolius) sobre los niveles de glucosa en ratones con diabetes mellitus tipo II inducidos con Aloxano.

\section{MATERIALES Y MÉTODOS}

Diseño y tipo de investigación. El estudio es de tipo longitudinal de diseño experimental (Hernández, Fernández, \& Baptista, 2010)

Muestra. Estuvo conformada por 42 ratones albinos de 2 meses de edad entre 30-40g de peso, inducidos a DMT2 y divididos en 2 grupos experimentales control y tratamiento. De los cuales 2 fallecieron

\section{Criterios de inclusión.}

- Ratones albinos machos y hembras con pesos entre 30 a 40 gr.

- Ratones albinos con DMT2 inducidos con aloxano.

- Ratones con buen estado de salud.

Criterios de exclusión.

- Se excluyeron ratones albinos que no cambiaron sus niveles de glucosa.

- Ratones que presenten alguna patología.

- $\quad$ Ratones < 30gr y > a 40gr de peso.

Análisis bioquímico. Para determinar los niveles de glucosa, se utilizó el glucómetro. La evaluación de glucosa se realizó en ayunas, para el grupo de control y tratamiento. Se tomó los valores de la glucosa basal. Después de inducir con aloxano a DM2 de la glucosa inducida al iniciar el tratamiento. El mismo procedimiento se realizó al finalizar el mismo. 
Selección, racionamiento y administración del yacón. Las raíces del yacón fueron compradas en el mercado de Puerto Nuevo - Ñaña. Fueron lavadas y cortadas en pequeños trozos, luego se envasaron en bolsas de polietileno. Las raíces fueron administradas en dos periodos: $50 \mathrm{~g}$ en la mañana y $50 \mathrm{~g}$ por la tarde, por un mes, se les monitorizó todos los días para ver el consumo del yacón.

Procesamiento y análisis de datos: Para el análisis estadístico se usó el programa estadístico SPSS, (versión 23), con el propósito de organizar la información en una base de datos, para luego realizar el procesamiento estadístico de los mismos. Se realizó la descripción de las variables de estudio mediante las siguientes medidas estadísticas: la media y la desviación estándar.
Para responder a la hipótesis planteada en el capítulo II, se determinó mediante la prueba estadística del análisis de varianza de un solo factor y la U de MannWitney (de acuerdo al análisis de normalidad).

Consideraciones éticas del uso de animales de laboratorio. De acuerdo con la ética de experimentación con animales de laboratorio, el respeto por la vida, el dolor o el sufrimiento a que estos puedan ser sometidos, se tomó en cuenta los criterios: exigencia de la norma sobre el uso de animales en actividades de investigación, no se han reportado efectos adversos sobre el consumo de $100 \mathrm{~g}$ de yácon por lo que no se consideró peligroso brindar esta dosis a los animales.

\section{RESULTADOS}

Tabla 1

Descripción del nivel de glucosa en ratones albinos inducidos a diabetes mellitus 2 (Aloxano) antes, durante y después en el grupo control y tratamiento yácon (smallanthus sonchifolius)

\begin{tabular}{lllllll}
\hline Grupo de experimental & & & \multicolumn{3}{c}{ Grupo control } \\
\hline & $\begin{array}{l}\text { Glucosa } \\
\text { Inicial }\end{array}$ & $\begin{array}{l}\text { Glucosa } \\
\text { Media }\end{array}$ & $\begin{array}{l}\text { Glucosa } \\
\text { Final }\end{array}$ & $\begin{array}{l}\text { Glucosa } \\
\text { Inicial }\end{array}$ & $\begin{array}{l}\text { Glucosa } \\
\text { Media }\end{array}$ & $\begin{array}{l}\text { Glucosa } \\
\text { Final }\end{array}$ \\
\cline { 2 - 7 } Media & 135.30 & 121.85 & 107.70 & 130.60 & 141.05 & 151.45 \\
Desv. típ. & 8.76 & 6.62 & 3.59 & 12.31 & 15.09 & 18.67 \\
Mínimo & 120.00 & 114.00 & 100.00 & 115.00 & 127.00 & 134.00 \\
Máximo & 150.00 & 132.00 & 115.00 & 169.00 & 186.00 & 204.00 \\
N & 20 & 20 & 20 & 20 & 20 & 20 \\
\hline
\end{tabular}

En la tabla 1, se observa que el promedio de glucosa inicial en el grupo experimental es menor que el grupo control por otro lado en el grupo experimental, el promedio de glucosa inicial es de $135.30 \mathrm{mg} / \mathrm{dl}$, mientras que en el grupo control es de $130.60 \mathrm{mg} / \mathrm{dl}$, siendo la variabilidad de ambos grupos 8.76 y 12.31 $\mathrm{mg} / \mathrm{dl}$. Individualmente mostrándose que la glucosa experimental es menor con respecto al grupo control.
Este resultado es similar al finalizar el tratamiento, ya que el promedio de glucosa final fue de $107.70 \mathrm{mg} /$ dl en el grupo experimental y $151.45 \mathrm{mg} / \mathrm{dl}$ en el grupo control, siendo la varianza de 3.59 y 18.67 correspondiente. Se demostró que el consumo de yacón durante 34 días logra reducir los niveles de glicemia.

Tabla 2

Análisis de varianza de glucosa en ratones albinos con DM2, según el tratamiento y control en tres fases* de evaluación durante el tratamiento.

\begin{tabular}{lllllll}
\hline Efecto de la glucosa & & Valor & $\mathrm{F}$ & $\mathrm{GL}$ & GL del error & $\mathrm{P}$ \\
\hline \multirow{3}{*}{ Grupo control } & Traza de Pillai & .86 & 56.87 & 2.00 & 18.00 & .00 \\
& Lambda de Wilks & .14 & 56.87 & 2.00 & 18.00 & .00 \\
& Traza de Hotelling & 6.32 & 56.87 & 2.00 & 18.00 & .00 \\
Grupo con & Traza de Pillai & .90 & 84.27 & 2.00 & 18.00 & .00 \\
tratamiento & Lambda de Wilks & .09 & 84.27 & 2.00 & 18.00 & .00 \\
& & & & & & \\
\hline *Evaluación inicial intermedia frinal & & & & & & \\
\end{tabular}

*Evaluación inicial intermedia y final 
En la Tabla 2, se observa según el análisis de varianza de un solo factor y un nivel de confianza del $99 \%$, que existe diferencia estadísticamente significativa entre el promedio de la glucosa de la inicial, intermedia y final, en los dos grupos referido a $(\mathrm{p}<.01)$.

Tabla 3

Comparar la glucosa entre el grupo experimental y control antes, durante y después en la fase del tratamiento.

\begin{tabular}{|c|c|c|c|c|c|}
\hline & Grupo & Rango promedio & U de Mann- Whitney & Z & $p$ \\
\hline \multirow[t]{3}{*}{ Antes } & Experimental & 24.1 & 128 & -1.96 & 0.06 \\
\hline & Control & 16.9 & & & \\
\hline & Experimental & 11.75 & 25 & -4.74 & 0 \\
\hline \multirow[t]{2}{*}{ Durante } & Control & 29.25 & & & \\
\hline & Experimental & 10.5 & 0 & -5.424 & 0 \\
\hline Después & Control & 30.5 & & & \\
\hline
\end{tabular}

En la tabla 3 se aprecia, según la prueba estadística U de Mann-Whitney y a un nivel de confianza al 99\%, que no existe diferencia estadísticamente significativa de la glucosa entre grupo experimental y control antes del tratamiento $(\mathrm{p}>.01)$.
Asimismo se evidencia que el nivel de glicemia después del consumo de yacón ha disminuido significativamente en $(p<.01)$.
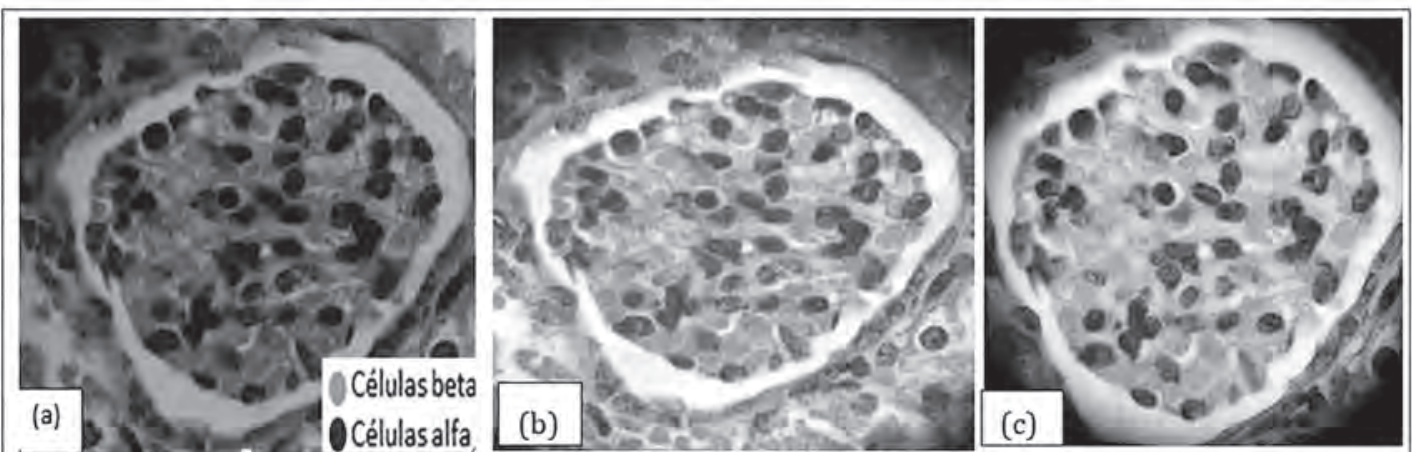

Figura 1: Corte histológico sano del páncreas con tinción (H\&E), Micrografías tomadas a $400 \mathrm{X}$ y 18 pixeles, se observa las células de los islotes de Langerhans, alfa y beta sin alteración en su estructura morfológica. a) Forma perimétrica regular de los islotes b) distribución regular de las células beta y alfa en el islote c) morfología simétrica celular.
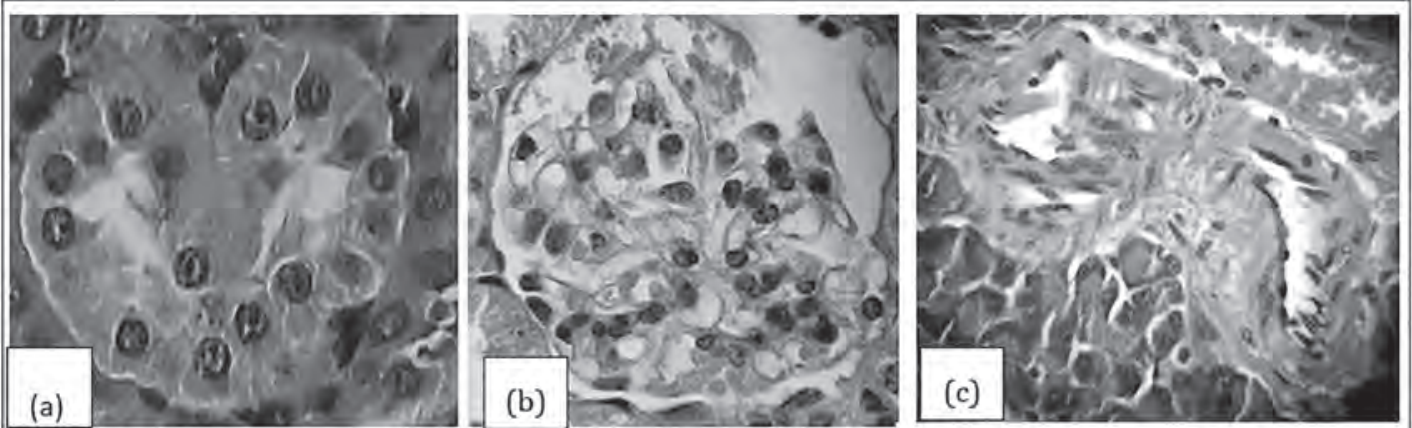

Figura 2: Islote de Langerhans con perímetro reducido al término de 36 días de inducción, con Aloxano en ratones diabéticos 2. a) Disminución de la población de células alfa y beta, b) desorden en la distribución celular en el islote de Langerhans, c) deformación del islote de Langerhans y nulidad de las células productoras de insulina. 


\section{DISCUSIÓN}

El control de la glicemia en la diabetes mellitus tipo II, es importante y vital para una mejor calidad de vida (Ramírez M, Ascanio M, Coronado M, Goméz C, Mayorga L, 2011) (Pebes A., 2014). Los cuadros que desarrollan hiperglicemia consecuentemente incrementan el riesgo de sufrir ceguera, complicación renal, enfermedades cardiovasculares (Sanders, 2004).

La alimentación cumple un papel muy importante, que implica procesos digestivos que pasan por diferentes niveles de glucemia, los cuales pueden desencadenar complicaciones patológicas sobre el metabolismo de la glucosa. Existen alimentos que tienen propiedades hipoglucemiantes que estabilizan los niveles de glucosa en sangre, entre estos tenemos la raíz del yacón (Valentová, 2003), este es un fruto natural que contienen muchos beneficios para la salud, por tener gran parte de sus propiedades hipoglucemiantes por su alto contenido de (FOS) fructooligosacáridos (Oliveira et al., 2013) (Ziaroyska, Fernández, Millela, 2013).

El FOS es un tipo de azúcar especial que contiene esta raíz el cual no es metabolizado por el organismo ya que carece de enzimas digestivas necesarias, para su absorción, además aporta bajas calorías que pueden ser incluidas en la dieta de personas diabéticas (Tapia \& Fries, 2007) (Chesquibol et al., 2002) (Satoh, Audrey, \& Kudoh, 2013). Algunos estudios reportan que el consumo de esta raíz fresca reduce los niveles de glucosa en la sangre (Jimenez, 2011).

En el estudio la unidad de tratamiento fueron ratones albinos, a quienes se aplicó método químico mediante el fármaco Aloxano (monohidrato), con la finalidad de provocar supresión endocrina del páncreas (DM2) a nivel hormonal (células alfa y beta de los islotes Langerhans) y morfológico con destrucción o lesión de otros órganos como el hígado y riñón, como agente inductor diabetogénico estos han sido sintéticamente empleados para inducir diabetes en los animales (Zanoello, Melazzo, Kerpel, Filappi, 2002) (Cárdenas, 2008).

Investigaciones similares muestran la acción de Aloxano con amplia variación y vía de administración en la inducción de DM2, experimentadas en ratas y ratones, por vía intraperitoneal, donde Dipa et al, aplicaron Aloxano (150mg/kg) (Dipa, 2014).

En forma similar Escobedo (2015). Utilizaron 150 $\mathrm{mg} / \mathrm{Kg}$ cada 48 horas, por vía intraperitoneal cada $24 \mathrm{~h}$ en tres días. En el estudio se usó dosis progresivas de Aloxano. Con diluciones de 0,5 gr de Aloxano en volúmenes de 500 y $200 \mathrm{ml}$ de agua destilada, los cuales fueron administrados en dosis progresivos entre 0.1 a $0.7 \mathrm{ml}$, dos veces por semana por un tiempo de 36 días por vía intraperitoneal.

Al analizar los resultados de la inducción para DM2 en ratones albinos en dos grupos experimental y control se utilizó 100 gr de raíz de yacón en estado natural, administrados en dos raciones por día en la mañana y en la tarde.

En nuestro estudio realizado según los resultados de Aloxano y la glicemia, en la desviación estándar tanto para el grupo con tratamiento y el control los resultados son como siguen, que la glucosa sérica promedio, antes de la intervención en ratones albinos fue de $135.30 \mathrm{mg} / \mathrm{dl}$, (DS=8.76) y la intermedia fue de $121.85 \mathrm{mg} / \mathrm{dl}$, (DS=6.62) la final fue de $107.70 \mathrm{mg} /$ dl, (DS=3.59), esto indica que el consumo del yacón podría disminuir en un $27.6 \mathrm{mg} / \mathrm{dl}$, la glicemia en ratas con DM2.

Mientras que el grupo control inducido sin tratamiento se observó que los niveles de glucosa sérica fueron los siguientes: inicial fue $130.60 \mathrm{ml} / \mathrm{dl}$, (DS=12.31); la intermedia fue $141.05 \mathrm{mg} / \mathrm{dl}$, (DS=15.09) y la final $151.45 \mathrm{mg} / \mathrm{dl}, \quad(\mathrm{DS}=18.67)$; incrementando $20.85 \mathrm{ml} / \mathrm{dl}$ de la glucosa sérica, podemos ver que no existiendo una diferencia significativa donde los animales presentan características fenotípicas como: estado de ánimos disminuido, perdida de pelaje, orina mal oliente, disminución del apetito e ingesta de alta dosis de agua, esto es consecuencia de la enfermedad que según el estudio citológico presentan deterioros de órganos y células.

En la prueba de la U de Mann-Whitney los resultados previos de las evaluaciones, no se encontró ningún nivel de significancia por ser datos basales, esto es según el límite de confianza al 99\%, no existe diferencia estadísticamente significativa de la glucosa entre el grupo experimental y control antes del tratamiento $(\mathrm{p}>.01)$. Asimismo se evidencia que al final del tratamiento el nivel de confianza al $99 \%$, si existía diferencia estadísticamente significativa entre la mediana de estos dos grupos de la glucosa sérica con respecto al valor tabulado de $(\mathrm{p}<.01)$.

Según el estudio de Mayta et al. (2004). Encontró que la administración de $300 \mathrm{~g}$ de raíz fresca de yacón por vía oral, reduce la respuesta glicémica postprandial en sujetos sanos en 79.8\% ( $p=0.001)$ en el grupo tratado con yacón. Asimismo Ludeña, Rejavinsky y Belsuzarri. (Ludeña, Rejavinsky, \& Belsuzarri, 2004); en la aplicación de suplementación con 500g/ 
de raíz de yacón por un periodo de 90 días, en 30 sujetos con DM2, demostraron la reducción de $\mathrm{Hb}$ glicosilada en $1,98 \%$.

Por otro lado, Ornelas et al (2013), demostraron el efecto hipoglucemiante del extracto de raíz de yacón en ratas diabéticas. La muestra estuvo conformada por 4 grupos experimentales como el grupo control, el grupo que recibió el extracto de raíz de yacón, el grupo diabético no tratado (DM1) y el diabético tratado con el extracto de la raíz de yacón (Y-DM1). En sus resultado se encontró que una disminución del $38.36 \%$ de los niveles de glucosa en el grupo Y-DM1 en comparación al grupo DM1 $(\mathrm{p}<0.05)$.

También Tasayco (2007), aplicó el uso de las hojas del yacón (Smallantus sonchifolius) como extracto hidroalcohólico al $10 \%$ p/v y demostró que podría aumentar la actividad hipoglucemiante en ratas con DM2. Se observó que el extracto de las hojas de yacón redujo los niveles de glucosa en un $(\mathrm{p}<0.001)$ en los grupos tratados con yacón y glibenclamida, también se demostró que hubo cambios en los marcadores toxicológicos bioquímicos ( $\mathrm{p}>0.05)$.

Finalmente, Habib, et al, (2011), confirmaron el efecto hipoglicemiante de la raíz del yacón en ratas. Los grupos experimentales fueron: grupo control con diabetes (DC), grupo control no diabéticos (ND), grupo diabéticos tratados con yacón dosis 1 (340mg FOS/kg peso, DT340) y grupo diabéticos tratados con yacón dosis 2 (6800mg FOS/kg peso, DT6800). Los resultados demostraron disminución de la glucosa para ambos grupos (DT340, DT6800) en comparación al grupo DC. También se muestra un aumento de la producción de insulina pancreática y glucagón like peptide-1. Estos cambios positivos se le atribuyen a los fructooligosacáridos del extracto de la raíz del yacón que podría ayudar a los pacientes con diabetes.

Estas investigaciones realizadas en las hojas y raíz del yacón corroboran con los resultados de nuestro estudio, demostrando que existe una disminución en los niveles de glucosa en sangre.

La DM2, tiene como característica común a la hipertrigliceridemia, debido a una disminución y deterioro progresivo de la masa de las células $\beta$ en los islotes de Langerhans o una disminución en la eficacia de los mecanismos de señalización (Olvera C, 2008). Esta destrucción ocurre en la masa de las células beta (CB) que su porcentaje es de $2 \%$ en la DM1, a diferencia de 40 a $60 \%$ que queda en la DM2. Según el estudio organográfico físicamente se observó que el órgano del páncreas fue deteriorado con una apariencia gelatinosa, el cual presentó una deformación en su forma anatómica, esto es debido a la acción citotóxica del aloxano. En la histología microscópica de este órgano se observó el deterioro de las células beta que es la principal fuente de producción de insulina que controla la glucosa en sangre, deformación de los islotes de Langerhans que componen al páncreas y deformación celular del tejido en estudio. Esto ocurre por la acción citotóxica del aloxano como agente inductor. En este estudio se confirma la efectividad del consumo de yacón sobre los niveles de glicemias en ratones albinos con DM2.

En conclusión, se encontró que el consumo de 100g/ día de yacón (Smallanthus sonchifolius) por un mes redujo significativamente el nivel de glucosa, es decir existe diferencia estadísticamente significativa en $(\mathrm{p}<.01)$.

Finalmente, se recomienda realizar estudios sobre el efecto hipoglucemiante del yacón utilizando un diseño experimental puro. También considerando el género de los animales para ver las respuestas a la inducción de DMT2 con la raíz del yacón.

\section{Declaración de financiamiento y de conflicto de intereses:}

El estudio fue financiado por los autores, quienes declaran no tener algún tipo de conflicto de interés en la investigación realizada.

\section{Correspondencia:}

América Mejía Vásquez.

Área de Producción, Clínica Good Hope, Malecón Balta, 956, Miraflores, Lima-Perú e-mail:america.mejia@goodhope.org.pe; americamejia21@hotmail.com 


\section{REFERENCIAS BIBLIOGRÁFICAS}

Aguilar C, Loreto M, A. O. (2013). Guias ALAD Sobre el control y tratamiento de la Diabetes Mellitus Tipo 2 con Medicina Basada en Evidencia.

Aschener P, F. N. (2013). Diabetes Atlas. Bruselas, Bélgica: Federación Internacional de la Diabetes.

Bash, E. (2015). Instituto Nacional de Estadística e Informática del Perú. Enfermedades no transmisibles y transmisibles. (Vol. 1). Lima.

Cárdenas, E. (2008). Química y evaluación del efecto hipoglicemiante de propólis en ratones diabeticos inducidos con aloxano. Chile.

Carla, A., Biazon, B., Marques, M., Wendt, N., Moreira, J. R., Vizioli, C., Amado, B. (2016). The in Vitro Antioxidant Capacities of Hydroalcoholic Extracts from Roots and Leaves of Yacon Do Not Correlate with Their in Vivo Antioxidant Action in Diabetic Rats. Scientific Research Publishing, 4(15), 13.

Chesquibol, N., Aguirre, R., Bravo, M., Lengua, R., Tomas, G., Delman, I., \& Rivera, D. (2002). Estudio químico y nutricional de las variedades de la raíz de la polymnia sonchifolia “yacon”. Revista Peruana de Química e Ingeniería Química, 5(1), 42.

Claude, J. (2011). Plan mundial contra la diabetes 20112121. Federación Internacional de Diabetes.

Dipa I, P. E. (2014). Comparative Study between the Effect of Momordica charantia (Wild Type) Fruits and Coccinia cordifolia's Leaf on Hypoglycemic and Hypolipidemic Activities of Alloxan Induced Type 2 Diabetic LongEvans Rats.

Escobedo G. (2015). Actividad Hipoglucemiante de hojas de Bauhinia variegata L. Rev Med. Mexico.

Ferreiras S, Azevedo C, Vardanega P, G. R. (2013). Antihiperglycemic effect of Quassia amara (Simaroubaceae ) in normal and diabetic rats. Rev. Bras. Pl. Med., 15(3), 5.

Gonzales N, Rodríguez E, M. H. (2013). Características Clinicas y Factores Asociados a morbilidad intrahospitalaria en los pacientes Mellitus Tipo 2. Rev. soc. Peru, 26(4), 7.

Gonzales R, C. N. (2000). Características clínicas de la Diabetes Mellitus en un área de Salud. Revista Cubana, 16(2), 6.

Gordillo, rocha, G. (2009). Efecto hipoglicemiante del extracto acuoso de las hojas Yacón en pacientes con Diabetes Mellitus Tipo 2. Tesis.

Habib N, Honoré s, Genta S, S. S. (2011). Hypolipidemic effect of yacon roots on diabetic rats: Biochemical approach. Chemico-Biological Interactions, 194(1), 9.

Hernández, R., Fernández, C., \& Baptista, M. del P. (2010). Metodología de la Investigación. (J. Mares, Ed.) (5ed.). Buenos Aires: McGraw-Hill.

Hirst M, Williams R, S. D. (2014). Diabetes Atlas. Bruselas, Bélgica. Federación Internacional de la Diabetes. Diabetes Voice, 59(3), 76. Isla, P. (2012). Artículo Original Diabetes Mellitus: La Pandemia del Siglo XXI. Revista Científica de Enfermería, 5(2), 13.

Jiménez K. (2011). Propuesta para el cultivo y aprovechamiento sostenible del yacón (Smallanthus sonchifolius (Poepp. \& Endl) H. Rob). Colombia.
Jurupe H; Herencia V; Robles E. (2007). Contribución al estudio de la actividad hipoglicemiante del yacón. Revista de La Facultad de Medicina, 7(2), 9.

Ludeña, A., Rejavinsky, K., \& Belsuzarri, I. (2004). Efectos normoglicemiante del tubérculo y la hoja del yacón (Smallantus Sonchifolius) en pacientes diabeticos tipo 2. Revista Horizonte Medico. México.

Mayta P, P. J. (2004). Reducción de la respuesta glicémica posprandial post-ingesta de raíz fresca de yacón en sujetos sanos. Artic. Orig. CIMEL., 9(1), 11.

Oliveira, R. B., Chagas-Paula, D. A., Secatto, A., Gasparoto, T. H., Faccioli, L. H., Campanelli, A. P., \& da Costa, F. B. (2013). Topical anti-inflammatory activity of yacon leaf extracts. Brazilian Journal of Pharmacognosy, 23(3), 9.

Olvera C, E. L. (2008). Páncreas y células beta: mecanismos de diferenciación, morfogénesis y especificación celular endocrina. ¿Regeneración? Tema Pediátrico, 65(1), 19.

Ornelas G, Pereira C, H. A. (2013). Improvement of biochemical parameters in tipo 1 diabetic rats after the roots aqueous extract of yacon (Poepp.\& Endl) treatment. Food and Chemical Toxicology, 59(1), 5.

Pebes A. (2014). Calidad de vida del paciente con Diabetes Mellitus Tipo 2 y control preventivo de la enfermedad en el Hospital Regional De Ica, 2010. Rev. Enferm Vanguard, 2(1), 6.

Portugal W, O. J. (2015). Diabetes Mellitus en el Siglo XXI. Dirección Regional de Salud del Callao Oficina de Epidemiología.

Ramírez M, Ascanio M, Coronado M, Goméz C, Mayorga L, M. R. (2011). Estilo de vida actual de los pacientes con diabetes mellitus tipo 2. Revista Ciencia y Cuidado, 8(1), 8.

Sanders, D. (2004). Lo Escencial en Sistema Endocrino y Aparato Reproductor. (E. S.A, Ed.) (Eegunda Ed). España.

Satoh, H., Audrey, M., \& Kudoh, A. (2013). Yacón diet (Smallanthus sonchofolius, Asteraceae) improves hepatic insulin resistance via reducing Trb3 expression in Zucker fa/fa rats. Nutrition \& Diabetes, 3(5), 6.

T. Yasayco. (2007). Actividad hipoglucemiante del extracto hidroalcohólico de las hojas de Smallanthus sonchifolius (yacón) en ratas con diabetes tipo 1 y 2. Tesis de Grado, 68.

Tapia, M. E., \& Fries, A. M. (2007). Guía de campo de los cultivos andinos. Fao; Anpe-Perú.

Valentová K, U. J. (2003). Smallanthus sonchifolius and Lepidium meyenii - prospective Andean crops for the prevention of chronic diseases. Biomedical Papers of the Medical Faculty of the University Palack., 147(2), 12. http://doi.org/10.5507/bp.2003.017

Zanoello A, Melazzo C, Kerpel J, Filappi A, P. D. (2002). Efeito protetor do syzygium cumini contra diabetes mellitus induzido por aloxano em ratos. Acta Farmaceutica Bonaerense, 21(1), 31-35.

Ziaroyska J, Fernandez E, Millela L, S. N. (2013). A Revised Its Nucleotide Sequence Gives ASpecifity For Smallanthus Sonchifolius (Poepp. and Endl) And It's Products Identificacion, 45(1), 10. http://doi.org/0.2298/ GENSR1301075M 